\title{
The Dirac Equation with the Scattered Electron Including Extra Potential Energy Comes from the Virial Theorem
}

\author{
Hasan Arslan \\ Physics Department, Bingöl University, Bingöl, Turkey \\ Email: hasanarslan46@yahoo.com
}

Received January 29, 2013; revised February 28, 2013; accepted March 9, 2013

Copyright (C) 2013 Hasan Arslan. This is an open access article distributed under the Creative Commons Attribution License, which permits unrestricted use, distribution, and reproduction in any medium, provided the original work is properly cited.

\begin{abstract}
The scattering of electron by a photon is a well-known reaction in physics. In this study, the change in the electron's energy after the scattering is taken into account. The previous works are searched. In order to take into account this change in the electron's energy in the equation of motion of the electron, the Dirac equation is used with the virial theorem. The scattered electron kinetic energy which is given to the electron by the loss in photon's energy is related to the potential energy of the electron by the virial theorem which states that the potential energy is two times of the kinetic energy in minus sign. A first time application of the virial theorem on a scattered electron by a photon is included to the Dirac equation.
\end{abstract}

Keywords: Dirac Equation; Virial Theorem; Scattered Electron

\section{Introduction}

The Dirac equation, virial theorem and photoelectric effect are searched from the previous works. The calculations needed for our purpose are done. The paper is designed as follows. In Section 2, the calculations are given. In Section 3, the results of these calculations are concluded.

\section{Calculations}

The scattered electron by a photon is simply given by the reaction

$$
e^{-}+\gamma \rightarrow \gamma+e^{-}
$$

The photon initially has an energy of $h v$ and after the reaction it has an energy of $h v^{\prime}$. The loss in photon's energy is the gain in the electron's kinetic energy. Therefore, the gained kinetic energy of the electron can be written as

$$
K=h\left(v-v^{\prime}\right)
$$

This process is widely defined in [1]. Here, we consider an electron in the electromagnetic interaction before scattering.

The Dirac equation for a particle interacting with an elecromagnetic field is derived in $[2,3]$ as

$$
\mathrm{i} \hbar \frac{\partial \psi}{\partial t}=\left(c \boldsymbol{\alpha} \cdot\left(\boldsymbol{p}-\frac{e}{c} \boldsymbol{A}\right)+\beta m c^{2}+e \phi\right) \psi
$$

where,

$$
\beta=\left(\begin{array}{cc}
1 & 0 \\
0 & -1
\end{array}\right), \alpha=\left(\begin{array}{ll}
0 & \sigma \\
\sigma & 0
\end{array}\right)
$$

and $\sigma$ are the $2 \times 2$ Pauli matrices. By using the virial theorem, we can add to the Equation (3) an extra potential energy term given to the electron by the photon. Since the Equation (3) describes the electron before scattering we must add the potential energy term after scattering. The kinetic energy given in the Equation (2) is half of the potential energy of the scattered electron due to the virial theorem [4-6]. So, we denote the potential energy lost by the electron after scattering as

$$
V=-2 K=-2 h\left(v-v^{\prime}\right)
$$

Then, the Dirac equation given by the Equation (3) can be written as

$$
\mathrm{i} \hbar \frac{\partial \psi}{\partial t}=\left(c \boldsymbol{\alpha} \cdot\left(\boldsymbol{p}-\frac{e}{c}\right)+\beta m c^{2}+e \phi-4 \hbar \pi\left(v-v^{\prime}\right)\right)
$$

The wave function $\psi$ can be written in two-compo- 
nent [2] as

$$
\psi=\mathrm{e}^{-i m c^{2} t / \hbar}\left(\begin{array}{l}
\varphi \\
\chi
\end{array}\right)
$$

Putting Equation (7) in Equation (6) we get the equation

$$
\begin{aligned}
\mathrm{i} \hbar \frac{\partial}{\partial t}\left(\begin{array}{l}
\varphi \\
\chi
\end{array}\right)= & c \boldsymbol{\sigma} \cdot\left(\boldsymbol{p}-\frac{e}{c} \boldsymbol{A}\right)\left(\begin{array}{l}
\chi \\
\varphi
\end{array}\right)-2 m c^{2}\left(\begin{array}{l}
0 \\
\chi
\end{array}\right) \\
& +\left(e \phi-4 \pi \hbar\left(v-v^{\prime}\right)\right)\left(\begin{array}{l}
\varphi \\
\chi
\end{array}\right)
\end{aligned}
$$

By writing the small component $\chi$ of the wave function in terms of the large component of the wave function $\varphi$ by the equation [2];

$$
\chi=\frac{\boldsymbol{\sigma} \cdot\left(\boldsymbol{p}-\frac{e}{c} \boldsymbol{A}\right)}{2 m c} \varphi
$$

and using the Pauli spin matrices identity [2]

$$
\boldsymbol{\sigma} \cdot \boldsymbol{a} \boldsymbol{\sigma} \cdot \boldsymbol{b}=\boldsymbol{a} \cdot \boldsymbol{b}+\mathrm{i} \boldsymbol{\sigma} \cdot \boldsymbol{a} \times \boldsymbol{b}
$$

also defining the terms

$$
\boldsymbol{B}=\boldsymbol{\nabla} \times \boldsymbol{A}, \boldsymbol{A}=\frac{1}{2} \boldsymbol{B} \times \boldsymbol{r}, \boldsymbol{L}=\boldsymbol{r} \times \boldsymbol{p} \text {, and } \boldsymbol{S}=\frac{1}{2} \hbar \boldsymbol{\sigma}
$$

we can write the Equation (8) into two equations after some calculations. The equation for an electron in the electromagnetic interaction is

$$
\mathrm{i} \hbar \frac{\partial \varphi}{\partial t}=\left[\frac{\boldsymbol{p}^{2}}{2 m}-\frac{e}{2 m c}(\boldsymbol{L}+2 \boldsymbol{S}) \cdot \boldsymbol{B}+e \phi-4 \pi \hbar\left(v-v^{\prime}\right)\right] \varphi
$$

where $\boldsymbol{B}$ is the weak uniform magnetic field, $\boldsymbol{L}$ is the orbital angular momentum, and $\boldsymbol{S}$ is the electron spin.

In [7], the interaction of a field with an electromagnetic potential for an electron is given by

$$
\gamma^{0} \hat{V}=-e \gamma^{\mu} A_{\mu}
$$

where

$$
\hat{V}=-e A^{0} I+e \boldsymbol{\alpha} \cdot \boldsymbol{A}
$$

here $I$ is a $4 \times 4$ unit matrix and $\gamma$ represents the Dirac matrices. The second term on the right of Equation (14) is written in the Equation (12). The first term on the right of this equation can be replaced instead of the term $e \phi$. Doing so we obtain

$$
\mathrm{i} \hbar \frac{\partial \varphi}{\partial t}=\left[\frac{\boldsymbol{p}^{2}}{2 m}-\frac{e}{2 m c}(\boldsymbol{L}+2 \boldsymbol{S}) \cdot \boldsymbol{B}-e A^{0} I-4 \pi \hbar\left(v-v^{\prime}\right)\right] \varphi
$$

In Equation (15), $\boldsymbol{p}$ is the momentum operator, $m$ is the mass of the electron, $e$ is the electron charge, $c$ is the speed of light, $\boldsymbol{L}$ is the angular momentum of the electron, $\boldsymbol{S}$ is the spin operator of the electron, $\boldsymbol{B}$ is the weak uniform magnetic field, $e A^{0}$ is the potential energy of the electron before scattering, $\hbar$ is the Planck's constant, and $v$ and $v^{\prime}$ are the initial and final frequencies of the photon respectively. By comparing this equation to the previous works, it can be seen that this equation is the Schrödinger-Pauli wave equation when the potential energy absence during scattering is added.

\section{Conclusion}

One approach is done in this study to combine the Dirac equation and the virial theorem. The electron scattered by a photon gains some kinetic energy and this kinetic energy is related to the loss in potential energy according to the virial theorem. This extra potential energy is added to the Dirac Equation to describe the motion of the particle.

\section{REFERENCES}

[1] A. Beiser, "Concepts of Modern Physics," 4th Edition, McGraw-Hill International Editions, New York, 1987.

[2] J. B. Bjorken and S. D. Drell, "Relativistic Quantum Mechanics," McGraw-Hill, New York, 1964.

[3] J. J. Sakurai, "Advanced Quantum Mechanics," AddisonWesley, Menlopark, 1967.

[4] J. B. Marion and S. T. Thornton, "Classical Mechanics of Particles and Systems," 3rd Edition, Harcourt Brace Jovanovich, New York, 1988.

[5] H. Goldstein, "Classical Mechanics," Addison-Wesley Publishing Company, Inc., Boston, 1974.

[6] M. Brack, "Virial Theorems for Relativistic Spin- $1 / 2$ and Spin-0 Particles," Physical Review D, Vol. 27, No. 8, 1983, pp. 1950-1953. doi:10.1103/PhysRevD.27.1950

[7] W. Greiner, S. Schramm and E. Stein, "Quantum Chromodynamics," 2nd Edition, Springer-Verlag Berlin Heidelberg, Berlin, Heidelberg, 2002. doi:10.1007/978-3-662-04707-1 